\title{
A GOOD DRUG MADE BETTER: THE FULVESTRANT DOSE RESPONSE STORY
}

John F.R. Robertson, ${ }^{1}$ Justin Lindemann, ${ }^{2}$ Sally Garnett, ${ }^{2}$ Elizabeth Anderson, ${ }^{3}$ Robert I. Nicholson, ${ }^{4}$ Irene Kuter, ${ }^{5}$ Julia M.W. Gee ${ }^{4}$

${ }^{1}$ Graduate Entry Medicine and Health School (GEMS), University of Nottingham, Derby, UK; ${ }^{2}$ AstraZeneca, Alderley Park, Macclesfield, UK; ${ }^{3}$ Formerly AstraZeneca, Alderley Park, Macclesfield, UK; ${ }^{4}$ Breast Cancer Molecular Pharmacology Group, School of Pharmacy and Pharmaceutical Sciences, Cardiff University, Cardiff, UK, ${ }^{5}$ Massachusetts General Hospital, Boston, MA, USA

Elizabeth Anderson is a current employee of Boehringer-Ingelheim RCV GmbH \& Co KG, Vienna, Austria

\section{Running title:}

The fulvestrant dose response story

\section{Funding Source}

AstraZeneca provided support for the conduct of the study, data collection and project management.

\section{Corresponding author:}

Professor John F.R. Robertson MD

Division of Medical Sciences \& Graduate Entry Medicine

School of Medicine 
Faculty of Medicine \& Health Sciences

University of Nottingham

Royal Derby Hospital Centre

Derby

DE22 3DT, UK

Tel.: +44 (0)1332 724881

Fax: +44 (0)1332 724880

E-mail: john.robertson@nottingham.ac.uk

Abstract word count: $250 / 250$

Manuscript word count: 4332 


\section{Conflict of Interest}

John F.R. Robertson has acted as a consultant on advisory boards for AstraZeneca and Bayer HealthCare, and received research funding from Amgen, AstraZeneca, Bayer HealthCare, and Novartis. He has received honoraria for speaking at symposia organized by AstraZeneca and GlaxoSmithKline. He has provided expert testimony on fulvestrant before the European Medicines Agency. Robert I. Nicholson and Julia M.W. Gee have received research funding from AstraZeneca. Irene Kuter has acted as a consultant to or in an advisory role to AstraZeneca. Justin Lindemann is an employee of AstraZeneca. Sally Garnett is an employee of AstraZeneca and holds stock ownership with AstraZeneca. Elizabeth Anderson is a former employee of AstraZeneca and holds stock ownership with AstraZeneca. 


\section{Abstract}

Sequential use of endocrine therapies remains the cornerstone of treatment for hormone receptor-positive advanced breast cancer, prior to use of cytotoxic chemotherapy for unresponsive disease. Fulvestrant is an estrogen receptor (ER) antagonist approved for treatment of postmenopausal women with ER+ advanced breast cancer following failure of prior antiestrogen therapy. Initially approved at a monthly dose of $250 \mathrm{mg}$, the recommended fulvestrant dose was revised to $500 \mathrm{mg}$ (500 mg/month plus $500 \mathrm{mg}$ on Day 14 of Month 1) following demonstration of improved progression-free survival versus fulvestrant $250 \mathrm{mg}$. We have reviewed the dose-dependent effects of fulvestrant, both from a retrospective combined analysis of dose-dependent reduction of tumor biomarkers in the pre-surgical setting (three previously reported studies: Study 18, NEWEST [Neoadjuvant Endocrine Therapy for Women with Estrogen-Sensitive Tumors] and Trial 57), and from a review of clinical studies for advanced breast cancer in postmenopausal women. Analysis of presurgical data revealed a consistent dose-dependent effect for fulvestrant on tumor biomarkers, with increasing fulvestrant dose resulting in greater reductions in ER, progesterone receptor and Ki67 labeling index. The dose-dependent biological effect corresponds with the dosedependent clinical efficacy observed in the treatment of advanced breast cancer following failure of prior antiestrogen therapy. Although it remains to be determined in a Phase III trial, cross-trial comparisons suggest a dose-dependent relationship for fulvestrant as first-line treatment for advanced breast cancer. Overall, biological and clinical data demonstrate a strong dose-dependent relationship for fulvestrant, supporting the efficacy benefit seen with fulvestrant $500 \mathrm{mg}$ over the $250 \mathrm{mg}$ dose.

Key words [not in the article or journal title]: advanced breast cancer; endocrine therapy; estrogen receptor; postmenopausal; tumor biomarkers 


\section{Introduction}

Endocrine therapies provide effective and well-tolerated treatments for postmenopausal women with hormone receptor-positive breast cancer (estrogen receptor-positive [ER+] and/or progesterone receptor-positive $[\mathrm{PgR}+])$, both in the adjuvant setting ${ }^{1}$ and for the treatment of advanced disease. ${ }^{2}$

Aromatase inhibitors (AIs), which block production of estrogen through their interaction with the estrogen-producing enzyme aromatase, have demonstrated increased efficacy compared with the ER antagonist tamoxifen in postmenopausal women as first-line endocrine treatment for ER+ advanced breast cancer, ${ }^{3-6}$ and as adjuvant therapy for postmenopausal women with

early breast cancer. ${ }^{7-9}$ As such, AIs are now considered the standard of care as adjuvant endocrine therapy for postmenopausal women with hormone receptor-positive breast cancer.

Fulvestrant, a 17ß-estradiol analog, is an ER antagonist that competes with endogenous estrogen for binding to the ER.$^{10}$ However, unlike tamoxifen, which exhibits partial estrogen agonist activity, fulvestrant has no recognized estrogenic effect. It is thought that this is due to the fact that upon binding to the ER, fulvestrant induces a conformational change, leading to degradation of the ER and complete inhibition of ER signaling in animal models. ${ }^{11}$

Unfortunately, resistance to endocrine therapy will eventually develop. While optimal sequencing of appropriate hormone therapies is the ideal approach, very few randomized controlled trials have directly compared the effects of changing the order in which two different agents are given. ${ }^{2}$ Furthermore, the paucity of data led the authors of a recent review to conclude that no definitive recommendations could be made regarding the sequencing of endocrine therapies in patients with advanced breast cancer, and that patients should receive the most efficacious treatment in that setting, whilst also considering specific side effect 
issues for that patient. ${ }^{2}$ Early pre-clinical data demonstrated a lack of cross-reactivity between fulvestrant and tamoxifen with fulvestrant inhibiting growth of tamoxifen-resistant tumors. ${ }^{12}$ Similarly in the clinical setting, many postmenopausal women with advanced breast cancer that responded to first-line fulvestrant remained responsive to further endocrine treatment. ${ }^{13,14}$ Furthermore, tumors that have responded to prior treatment with an antiestrogen ${ }^{15,16}$ or an $\mathrm{AI}^{17,18}$ may retain sensitivity to subsequent treatment with fulvestrant.

Pre-surgical studies provide the opportunity to perform a detailed analysis and comparison of biomarker expression and biomarker response to various experimental drug treatments. As an example, the selective estrogen receptor modulator tamoxifen was reported to increase PgR levels as a result of its partial estrogen agonist activity. ${ }^{19}$ However, down regulation of ER with fulvestrant leads to reduction in PgR protein levels through disruption of ER-dependent transcription of the PgR gene, as shown in a randomized comparison with tamoxifen, highlighting the distinct mechanisms of action of these two agents. ${ }^{20}$ Reduction in Ki67 expression, a nuclear antigen and marker of cell proliferation, is reported to correlate with treatment response to endocrine therapy in ER+ breast cancer, ${ }^{21}$ and Ki67 in short-term neoadjuvant studies has been shown to predict outcome in long-term adjuvant trials. ${ }^{22}$

Clinical efficacy of fulvestrant was demonstrated in postmenopausal women with advanced breast cancer that had progressed or recurred on prior antiestrogen therapy ${ }^{16,23,24}$ and was originally approved at a monthly dose of $250 \mathrm{mg}$. However, a dose-dependent effect was subsequently shown, with improved progression-free survival (PFS) for fulvestrant $500 \mathrm{mg}$ (500 mg/month intramuscular [i.m.] injection plus $500 \mathrm{mg}$ on Day 14 of Month 1) versus the $250 \mathrm{mg}$ dose. This led to approval of the $500 \mathrm{mg}$ dose for the treatment of postmenopausal women with ER+ advanced breast cancer following failure of prior antiestrogen therapy. ${ }^{25}$ 
This review investigates the dose-dependent effects of fulvestrant more broadly, both in terms of the reduction of tumor biomarkers in the pre-surgical setting, and in clinical efficacy for the treatment of breast cancer. 


\section{Biological rationale for a dose-response relationship for fulvestrant}

Dose-dependent reduction of tumor biomarkers following fulvestrant treatment was first demonstrated in a short-term pre-surgical study in postmenopausal women with primary breast cancer. ${ }^{26}$ Following daily injections of a short-acting formulation of fulvestrant, reductions in ER expression and Ki67 labeling index were greater in patients with ER+ breast cancer who received a fulvestrant $18 \mathrm{mg}$ daily injection compared with those who received a fulvestrant $6 \mathrm{mg}$ daily injection.

We now extend the study of dose dependency by presenting a retrospective analysis of tumor biomarker data, extracted from three previously reported pre-surgical studies over a fulvestrant dose range of 50-500 $\mathrm{mg}$, administered using the commercially available longacting formulation. Data from Study $18,{ }^{20}$ NEWEST (Neoadjuvant Endocrine Therapy for Women with Estrogen-Sensitive Tumors), ${ }^{27}$ and Trial $57^{28}$ were combined in this analysis.

\section{Study designs}

\section{Study 18}

Study 18 was a randomized, multicenter, partially blinded study that compared placebo, tamoxifen, fulvestrant $50 \mathrm{mg}$, fulvestrant $125 \mathrm{mg}$, and fulvestrant $250 \mathrm{mg}$ prior to surgery in postmenopausal women with previously untreated primary breast cancer. ${ }^{20}$ Patients received a single i.m. dose of fulvestrant $50 \mathrm{mg}, 125 \mathrm{mg}, 250 \mathrm{mg}$, or tamoxifen $20 \mathrm{mg}$ daily, or tamoxifen placebo daily for 14-21 days prior to surgery. Only data from patients whose tumors were $\mathrm{ER}+$ or $\mathrm{PgR}+$ have been included in the current analysis. Where patients had more than one tumor, baseline data from only the primary tumor were included. 
NEWEST

NEWEST (ClinicalTrials.gov identifier NCT0093002) was a randomized, multicenter, openlabel, Phase II study comparing fulvestrant $500 \mathrm{mg}(500 \mathrm{mg} / \mathrm{month}$ plus $500 \mathrm{mg}$ on Day 14 of Month 1) with fulvestrant $250 \mathrm{mg} / \mathrm{month}$ for 16 weeks prior to surgery in postmenopausal women with ER+ locally advanced breast cancer. ${ }^{27}$ Tumor biomarker levels at Week 4 have been used in the present analysis for the closest consistency with data from Study 18 and Trial 57.

\section{Trial 57}

Trial 57 (ClinicalTrials.gov identifier NCT00259090) was a randomized, multicenter, doubleblind, Phase II trial comparing fulvestrant $500 \mathrm{mg}$ (single i.m. dose) plus anastrozole (1 mg orally once daily for 14-21 days), or fulvestrant $500 \mathrm{mg}$ plus anastrozole placebo, or anastrozole plus fulvestrant placebo prior to surgery in postmenopausal women with ER+ primary breast cancer. ${ }^{28}$ Prior to protocol amendment, Trial 57 included a treatment phase in which patients were randomized to receive fulvestrant $250 \mathrm{mg}$ plus anastrozole $(\mathrm{n}=6)$, fulvestrant $250 \mathrm{mg}$ plus anastrozole placebo $(\mathrm{n}=6)$, or anastrozole $1 \mathrm{mg}$ plus fulvestrant placebo $(n=6)$. Although patient numbers are small and should therefore be interpreted with caution, data for this initial treatment phase have been included for completeness in this analysis.

\section{Tumor biomarker expression and statistical analyses}

$\mathrm{ER}, \mathrm{PgR}$ and Ki67 expression were determined in each study by immunochemistry on sections of formalin-fixed, paraffin-embedded tissue. Study 18 used the following antibodies: ER, H222 (Abbott); PgR, KD68 (Abbott); Ki67, MIB-1 (Coulter Electronics). In NEWEST, the antibodies used were: ER, 1D5 (Dako Ltd); PgR, 636 (Dako Ltd); Ki67, MIB-1 (Coulter Electronics). The antibodies used in Trial 57 were as follows: ER, 6F11 (Novocastra); PgR, 
636 (Dako Ltd); Ki67, Clone MIB-1 (Dako Ltd). Antigen retrieval methods and secondary detection methods varied between the studies and have been described previously. ${ }^{20,27,28} \mathrm{ER}$, PgR and Ki67 expression levels at pre- and post-treatment (14-21 days post-treatment in Study 18 and Trial 57 and at Week 4 in NEWEST) were determined by manual counting under light microscopy. ER and PgR expression were determined as the H-score, calculated as $(0.5 \times \%+/-)+(1 \times \%+)+(2 \times \%++)+(3 \times \%+++)$, where $\%+/-, \%+, \%++$, and $\%+++$ represent the overall percentage positivity of very weak, weak, moderate, and strong staining, respectively. Ki67 expression was determined as the labeling index, derived from the number of positively stained epithelial cells, expressed as a percentage of the total number of cells counted.

Tumor biomarker expression data were analyzed by study using an analysis of covariance (ANCOVA) model (log-transformed ratio of post- to pre-treatment) with the log-transformed baseline value and treatment included as factors. The least squares mean and confidence interval $(\mathrm{CI})$ values were back-transformed to the original scale. To assess the impact of fulvestrant dose while allowing for between-study variability, a second ANCOVA model was produced including log-transformed baseline, dose (as a continuous variable), and study as factors. The first ANCOVA included all treatment groups within each trial; the second ANCOVA included only placebo and the fulvestrant $50 \mathrm{mg}, 125 \mathrm{mg}, 250 \mathrm{mg}$, and $500 \mathrm{mg}$ treatment groups. In order for the placebo data to be log-transformed, a dose of $0.5 \mathrm{mg}$ rather than $0 \mathrm{mg}$ was used for the purpose of this analysis.

\section{ER H-score}

In Study 18, NEWEST, and Trial 57, a dose-dependent effect was seen over the dose ranges investigated for reduction in ER expression. In each study, the greatest reduction in ER expression was seen with the highest fulvestrant dose. In Study 18, greater reduction in ER 
was observed for fulvestrant $250 \mathrm{mg}$ versus tamoxifen, and in Trial 57, greater reduction in ER expression was observed for fulvestrant $500 \mathrm{mg}$ versus anastrozole. In Trial 57, no additional reduction in ER expression was observed for fulvestrant $500 \mathrm{mg}$ plus anastrozole compared with fulvestrant $500 \mathrm{mg}$ alone (Table 1; Figure 1).

\section{PgR H-score}

A consistent dose-dependent effect of fulvestrant was also observed in Study 18, NEWEST, and Trial 57 for reduction in $\mathrm{PgR}$ expression. The greatest reduction in $\mathrm{PgR}$ expression was seen with the highest fulvestrant dose within each study. An increase in PgR expression was seen in the tamoxifen treatment group in Study 18. In Trial 57, no additional reduction in PgR expression was observed for the combination of fulvestrant $500 \mathrm{mg}$ plus anastrozole compared with either fulvestrant $500 \mathrm{mg}$ alone or anastrozole alone. Similar reductions in PgR expression were observed for fulvestrant $500 \mathrm{mg}$ alone and anastrozole alone (Table 2; Figure 2).

\section{Ki67 labeling index}

Ki67 labeling index was reduced following treatment in each fulvestrant treatment group in each study. In Study 18 and NEWEST, the greatest reduction in Ki67 labeling index was seen with the highest fulvestrant dose. In Trial 57, which also included the small initial cohort of patients treated with fulvestrant $250 \mathrm{mg}(\mathrm{n}=6)$, there were no meaningful differences in Ki67 labeling index reduction between the fulvestrant treatment groups (Table 3; Figure 3).

Overall results from the ANCOVA model show a consistent dose-dependent effect for fulvestrant over the dose ranges analyzed for ER and PgR H-score and Ki67 labeling index. Results for the second ANCOVA, which adjusted for between-study variability, show that 
increasing fulvestrant dose results in greater reduction in ER and PgR H-score and Ki67 labeling index $(\mathrm{p}<0.0001$ for the dose-response relationship for each biomarker). 


\section{Clinical evidence of a dose-response relationship for fulvestrant}

\section{Fulvestrant dose-response in second-line therapy for advanced breast cancer}

The clinical efficacy of fulvestrant at a dose of $250 \mathrm{mg} / \mathrm{month}$ was established in the registration trials 0020 and 0021 , which compared fulvestrant $250 \mathrm{mg}$ with anastrozole for the treatment of postmenopausal women with advanced breast cancer that had progressed or recurred on prior antiestrogen therapy. ${ }^{23,24}$ In a combined analysis of data from both studies (fulvestrant, $n=428$; anastrozole, $n=423$ ), fulvestrant $250 \mathrm{mg}$ was shown to be at least as effective as anastrozole with respect to time to progression (TTP). Median TTP was 5.5 months for fulvestrant $250 \mathrm{mg}$ compared with 4.1 months for anastrozole (hazard ratio [HR] $0.95 ; 95.14 \%$ CI $0.82,1.10 ; \mathrm{p}=0.48) .{ }^{16}$ This led to the approval of fulvestrant $250 \mathrm{mg}$ for the treatment of postmenopausal women with advanced breast cancer that had progressed or recurred on prior antiestrogen therapy. However, evidence of dose-dependent clinical efficacy with fulvestrant had already been suggested in these studies, as an initial $125 \mathrm{mg}$ dose was dropped following a planned interim assessment that found no evidence for clinical efficacy at the fulvestrant $125 \mathrm{mg}$ dose. Given the favorable tolerability profile of fulvestrant $250 \mathrm{mg}$, alternative dosing regimens were investigated.

The Phase III CONFIRM (COmparisoN of Faslodex In Recurrent or Metastatic breast cancer) trial was designed to compare fulvestrant $500 \mathrm{mg}$ with fulvestrant $250 \mathrm{mg}$ in patients with hormone receptor-positive, pre-treated, advanced breast cancer. Fulvestrant $500 \mathrm{mg}$ significantly prolonged PFS versus fulvestrant $250 \mathrm{mg}$. Median PFS was 6.5 months in the fulvestrant $500 \mathrm{mg}$ group compared with 5.5 months in the fulvestrant $250 \mathrm{mg}$ group (HR 0.80; 95\% CI 0.68, 0.94; $\mathrm{p}=0.006$ ), demonstrating a clear dose-dependent relationship for fulvestrant in this setting (Table 4). ${ }^{25}$ Importantly, the dose-dependent clinical efficacy seen in CONFIRM was not associated with a dose-dependent increase in toxicity, with no 
substantial differences between the treatment groups in terms of incidence and severity of adverse events. This increase in therapeutic index led to fulvestrant $500 \mathrm{mg}$ becoming the recommended dose. This benefit was further confirmed in a follow-up analysis, performed when approximately $75 \%$ of patients had died. Median overall survival was 26.4 months for fulvestrant $500 \mathrm{mg}$ compared with 22.3 months for fulvestrant $250 \mathrm{mg}$, indicating a clinically relevant difference in overall survival between the treatment groups (HR 0.81; 95\% CI 0.69, 0.96; nominal $\mathrm{p}=0.016)^{29}$

\section{Fulvestrant dose-response in first-line therapy for advanced breast cancer}

Cross-trial comparisons also suggest a dose-response relationship for fulvestrant as first-line therapy for advanced breast cancer. In Trial 25, fulvestrant $250 \mathrm{mg}$ failed to demonstrate noninferiority compared with tamoxifen, the standard of care at the time of the trial, in postmenopausal women with advanced breast cancer previously untreated with endocrine therapy for advanced disease. ${ }^{30}$ As anastrozole was previously shown to demonstrate improvements in efficacy over tamoxifen, ${ }^{3}$ this was considered a surprising outcome for fulvestrant $250 \mathrm{mg}$. However, with the almost immediate separation of the TTP curves in this trial, it was hypothesized that the 3-6 months to steady state for the fulvestrant $250 \mathrm{mg}$ regimen could have led to the under-performance of this treatment group.

In the Phase II FIRST study (Fulvestrant fIRst-line Study comparing endocrine Treatments), fulvestrant $500 \mathrm{mg}$ was compared with anastrozole in postmenopausal women with advanced breast cancer who had not received endocrine therapy for advanced disease. The fulvestrant $500 \mathrm{mg}$ dose regimen, which includes a $500 \mathrm{mg}$ dose at Day 14, was shown to be at least as effective as anastrozole in terms of the primary endpoint of clinical benefit rate (fulvestrant, 72.5\%; anastrozole, 67.0\%) and the secondary endpoint of TTP was significantly longer for fulvestrant $500 \mathrm{mg}$ compared with anastrozole. ${ }^{31}$ Safety data indicated that fulvestrant 500 
$\mathrm{mg}$ has a similar tolerability profile compared with anastrozole $1 \mathrm{mg}$, and is well tolerated as first-line therapy for advanced breast cancer. In a follow-up analysis, which was performed when disease had progressed in approximately $75 \%$ of patients, median TTP was 23.4 months for fulvestrant $500 \mathrm{mg}$ compared with 13.1 months for anastrozole (hazard ratio $0.66 ; 95 \%$ CI $0.47,0.92 ; \mathrm{p}=0.01) .{ }^{14}$ This was the first trial to indicate that an alternative endocrine therapy may be more effective than an AI in the first-line setting for advanced breast cancer, and indirectly suggests a dose-response relationship for fulvestrant $500 \mathrm{mg}$ over fulvestrant $250 \mathrm{mg}$ as first-line therapy for advanced breast cancer. Given that fulvestrant $250 \mathrm{mg}$ demonstrated non-inferiority to anastrozole (in the second-line setting of the registration trials 0020 and $0021^{16,23,24}$ ), the significantly longer TTP with fulvestrant 500 mg versus anastrozole in the first-line setting was also indirect evidence of a dose-response relationship for fulvestrant.

\section{Fulvestrant dose-response in the neoadjuvant setting}

NEWEST was the first study to compare the biological and clinical activity of the fulvestrant $500 \mathrm{mg}$ dose regimen versus fulvestrant $250 \mathrm{mg}$. Whilst the primary endpoint of NEWEST was biological (change in Ki67 labeling index from baseline to Week 4), the clinical data appeared to correspond with the dose-dependent reduction in tumor biomarkers seen at Week 4. The tumor response rate at Week 4 was $17.4 \%$ for the fulvestrant $500 \mathrm{mg}$ group compared with $11.8 \%$ in the fulvestrant $250 \mathrm{mg}$ group (odds ratio [OR] 1.68; 95\% CI 0.77, 3.70; $\mathrm{p}=0.19$ ). At Week 16, tumor response was $22.9 \%$ in the fulvestrant $500 \mathrm{mg}$ group compared with $20.6 \%$ in the fulvestrant $250 \mathrm{mg}$ group (OR 1.30; 95\% CI 0.64, 2.64; $\mathrm{p}=0.47$ ). ${ }^{27}$ 


\section{Fulvestrant in combination therapy}

Together with its distinct mechanism of action and reduced risk of cross-resistance with other endocrine treatments, the observation of incomplete ER reduction with fulvestrant $250 \mathrm{mg}$, both in the short, ${ }^{20}$ medium, and long term ${ }^{32,33}$ led to combination therapies being developed, aiming to further reduce ER activity and improve efficacy. The FACT (Fulvestrant and Anastrozole Combination Therapy) study compared the efficacy of a combination of anastrozole plus the fulvestrant $250 \mathrm{mg}$ loading dose (LD) regimen (fulvestrant $250 \mathrm{mg}+$ LD: $500 \mathrm{mg}$ Day 0, $250 \mathrm{mg}$ Days 14 and 28, $250 \mathrm{mg} / \mathrm{month}$ thereafter) versus anastrozole alone as therapy for postmenopausal women at first relapse after primary treatment for localized hormone receptor-positive breast cancer. ${ }^{34}$ Over $60 \%$ of the participants had received a prior endocrine therapy in the adjuvant setting. For the primary endpoint, median TTP was 10.8 months in the fulvestrant $250 \mathrm{mg}+\mathrm{LD}$ plus anastrozole combination compared with 10.2 months in the anastrozole alone treatment group (HR 0.99; $95 \%$ CI 0.81, 1.20; $\mathrm{p}=0.91) .{ }^{34}$ The overall incidence of AEs was similar between the two treatment groups. Secondary endpoints including objective response rate, clinical benefit rate and overall survival were also similar between the two study arms, indicating no benefit for the anastrozole plus fulvestrant combination over anastrozole alone.

The randomized Phase III SWOG S0226 trial also compared the combination of anastrozole plus fulvestrant $250 \mathrm{mg}+\mathrm{LD}$ with anastrozole alone as first-line therapy for postmenopausal women with metastatic breast cancer. ${ }^{35}$ The primary endpoint of PFS was significant in favor of the combination group: 15.0 months compared with 13.5 months in the anastrozole alone group (HR 0.80; 95\% CI 0.68, 0.94; $\mathrm{p}=0.007$ ), and no safety concerns were raised with the fulvestrant plus anastrozole combination. In a retrospective analysis of those patients naïve to prior tamoxifen therapy (414/694 patients: $59.7 \%$ ), median PFS was 17.0 months in the 
combination group compared with 12.6 months in the anastrozole alone group (HR 0.74; 95\% CI 0.59, 0.92; $\mathrm{p}=0.006)$. In those patients who had received previous treatment with tamoxifen (280/694 patients: 40.3\%), median PFS was 13.5 months in the combination group compared with 14.1 months in the anastrozole alone group (HR 0.89; 95\% CI 0.69, 1.15; $\mathrm{p}=0.37) .{ }^{35}$ In total, 166 out of 514 patients $(32.3 \%)$ had received no prior adjuvant endocrine therapy in the FACT trial, whereas 414 out of 694 patients $(59.7 \%)$ were naïve to prior tamoxifen in SWOG S0226. In both trials, less than $2 \%$ of patients had received adjuvant therapy with an AI. As the percentage of patients who were naïve to prior adjuvant endocrine treatment was lower in the FACT trial, and any potential differences in clinical effectiveness may be more pronounced when comparing endocrine agents in hormone-naïve patients, this could provide one potential explanation for the differences in efficacy between the FACT and SWOG S0226 trials. Furthermore, initial data from the SoFEA trial failed to demonstrate improved efficacy for the combination of fulvestrant with an AI over a monotherapy treatment in the second-line setting. Similar PFS was reported for fulvestrant $250 \mathrm{mg}+\mathrm{LD}$ in combination with anastrozole compared with fulvestrant alone in postmenopausal patients with advanced breast cancer following progression on non-steroidal AIs. ${ }^{36}$ 


\section{Discussion}

Our analysis of fulvestrant in three pre-surgical studies demonstrates a strong dose-dependent biological effect in the reduction of tumor biomarkers. Across each dataset analyzed, increasing fulvestrant dose leads to increased reduction in ER, PgR and Ki67, and this dosedependent reduction in tumor biomarkers corresponds to the dose-dependent clinical efficacy seen in postmenopausal women with advanced breast cancer in the second-line setting of trials 0020 and 0021 (fulvestrant $250 \mathrm{mg}$ vs $125 \mathrm{mg}$ ) and CONFIRM (fulvestrant $500 \mathrm{mg}$ vs $250 \mathrm{mg}$ ). In the first-line setting, increased efficacy of fulvestrant $500 \mathrm{mg}$ (vs anastrozole in FIRST) versus $250 \mathrm{mg}$ (versus tamoxifen in Trial 25) has also been implied through indirect, cross-trial comparisons. Further cross-trial comparisons show that in the registration trials 0020 and 0021 (in the second-line setting), fulvestrant $250 \mathrm{mg}$ was non-inferior to anastrozole, but in the Phase II FIRST trial (fulvestrant $500 \mathrm{mg}$ vs anastrozole in the first-line setting), fulvestrant demonstrated significantly longer TTP. The ongoing Phase III FALCON (Fulvestrant and AnastrozoLe COmpared in hormonal therapy Naïve advanced breast cancer) trial (ClinicalTrials.gov identifier: NCT01602380) will provide the additional evidence required, for registration purposes, to determine if fulvestrant $500 \mathrm{mg}$ is a better option than anastrozole $1 \mathrm{mg}$ as first-line hormonal therapy for postmenopausal women with locally advanced or metastatic breast cancer.

Other than the SWOG S0226 trial, clinical data do not support the combination of an antiestrogen with an AI. While it is possible that the fulvestrant $250 \mathrm{mg}$ loading dose used in the FACT and SoFEA trials was sub-optimal and that efficacy might have been improved with the $500 \mathrm{mg}$ dose, biological data using fulvestrant $500 \mathrm{mg}$ do not suggest there will be an additional benefit by adding an AI to fulvestrant $500 \mathrm{mg} .{ }^{28}$ Unfortunately, the SWOG S0226 trial did not have a third treatment arm of fulvestrant $250 \mathrm{mg}$ alone, which would have 
clarified whether the longer TTP seen with the combination would have been achieved with fulvestrant alone in the first-line setting. The data from SWOG S0226 should therefore be used to generate hypotheses for studies to understand if and why this finding is real.

The dose-dependent effect on tumor biomarkers seen here in postmenopausal women may also be evident in pre-menopausal women receiving fulvestrant in clinical studies. In contrast to postmenopausal women, where fulvestrant $250 \mathrm{mg}$ was shown to be effective in reducing ER, PgR, and Ki67, a previous study demonstrated that fulvestrant $250 \mathrm{mg}$ did not effectively reduce expression of ER, PgR, or Ki67 in pre-menopausal women, who are known to have much higher levels of circulating estrogen which would be expected to compete with fulvestrant for binding to $\mathrm{ER} .^{37} \mathrm{~A}$ single fulvestrant dose of $750 \mathrm{mg}$ was subsequently shown to reduce ER levels in pre-menopausal women with primary breast cancer, to an extent similar to that seen with fulvestrant $250 \mathrm{mg}$ in postmenopausal women, suggesting a doseresponse relationship for fulvestrant in these patients. ${ }^{38}$

The dose-dependent biomarker data presented here also correspond to the previously-reported pharmacokinetic profile of fulvestrant. Following a single injection of either fulvestrant $50 \mathrm{mg}, 125 \mathrm{mg}$, or $250 \mathrm{mg}$, dose-linear pharmacokinetics were observed. ${ }^{39}$ In the NEWEST study, plasma levels and exposure with fulvestrant $500 \mathrm{mg}$ were approximately double that of fulvestrant $250 \mathrm{mg}$ at Week 16, demonstrating a linear dose-dependent effect. ${ }^{40}$

In addition to increasing the monthly fulvestrant dose in postmenopausal women, early pharmacokinetic and clinical data suggested that alternative regimens could be utilized to increase clinical efficacy. At the fulvestrant $250 \mathrm{mg} / \mathrm{month}$ dose, it takes $3-6$ months to achieve steady-state plasma levels. ${ }^{41}$ Therefore, alternative dose regimens were explored incorporating a loading dose component with the aim of reducing the time to reach steadystate and achieving earlier down regulation of ER. In EFECT (Evaluation of Faslodex versus 
Exemestane Clinical Trial), fulvestrant $250 \mathrm{mg}+\mathrm{LD}$ was employed (fulvestrant $250 \mathrm{mg}+$ LD: $500 \mathrm{mg}$ Day 0, $250 \mathrm{mg}$ Days 14 and 28, $250 \mathrm{mg} / \mathrm{month}$ thereafter) and the time required for fulvestrant to reach steady-state was reduced to approximately 1 month. ${ }^{18,42}$ Furthermore, the residual ER that remains detectable following treatment with fulvestrant $500 \mathrm{mg}$ in NEWEST and Trial $57^{27,28}$ raises the possibility that greater reductions in ER expression and improved efficacy could be achieved using an even higher fulvestrant dose. Fulvestrant 750 $\mathrm{mg}$, although requiring three separate $5 \mathrm{ml}$ intramuscular injections, has previously been shown to be well tolerated, and is effective in reducing ER expression in pre-menopausal women. ${ }^{38}$ However to our knowledge, no studies using a dose higher than fulvestrant $500 \mathrm{mg}$ for postmenopausal breast cancer have been reported, and three or more $5 \mathrm{~mL}$ injections per dose, as required by the current formulation, may present practical impediments. It should also be noted that if complete and irreversible elimination of ER expression could ever be achieved (thereby potentially making any subsequent endocrine therapy redundant), it would need to be established whether the total duration of control achieved by the total ER ablation was greater than sequential treatment with current endocrine therapies.

Trials other than those described above have also compared dose regimens of fulvestrant in postmenopausal women with advanced breast cancer. The Phase II FINDER (Faslodex InvestigatioN of Dose evaluation in Estrogen Receptor-positive advanced breast cancer) studies, conducted in Japan (FINDER1) and Europe (FINDER2) investigated the efficacy and safety of three dose regimens of fulvestrant (fulvestrant $500 \mathrm{mg}$; fulvestrant $250 \mathrm{mg}+\mathrm{LD}$; fulvestrant $250 \mathrm{mg}$ ) (Table 4). Although efficacy and safety were established for each of the fulvestrant doses in both studies, the relatively small sample sizes did not allow for any potential differences in efficacy to be determined between the treatment groups. ${ }^{43,44}$ 
Importantly, the biological and clinical studies analyzed here were not conducted concurrently, and indirect cross-trial comparisons should be interpreted with caution. Significant variability was observed between these studies both for absolute levels, and magnitude of treatment-driven change, of ER and PgR H-score and Ki67 labeling index. Factors that may have influenced this between-study variability include the different antibodies and antibody retrieval methods that were used in each of the studies, and the assay sensitivity, which was lower in the earlier Study 18 than NEWEST and Trial 57, where assay sensitivity was very high in order to reduce false-negative results. ${ }^{27}$ In addition, the time from dose to surgery or biopsy varied between the studies. However, when adjusting for betweenstudy variability in the analysis of tumor biomarker reduction using the second ANCOVA, fulvestrant demonstrated a strong dose-response relationship for reduction in ER, PgR, and Ki67 index.

\section{Conclusion}

Overall, the dose-response effect seen with fulvestrant in clinical studies in the advanced disease setting mirrors the dose-dependent reduction in tumor biomarker studies in a much earlier disease setting. Across all of the datasets analyzed here, a dose-response relationship has been demonstrated. This represents a strong demonstration of the relationship between the biology of tumor biomarkers and clinical efficacy which underpins rational drug development. 


\section{Acknowledgements}

We thank Simon Vass, $\mathrm{PhD}$ of Complete Medical Communications, who provided medical writing support, funded by AstraZeneca. 


\section{References}

1. Dowsett M, Cuzick J, Ingle J, et al. Meta-analysis of breast cancer outcomes in adjuvant trials of aromatase inhibitors versus tamoxifen. J Clin Oncol 2010; 28:509-18.

2. Barrios C, Forbes JF, Jonat W, et al. The sequential use of endocrine treatment for advanced breast cancer: where are we? Ann Oncol 2012; 23:1378-86.

3. Bonneterre J, Thürlimann B, Robertson JF, et al. Anastrozole versus tamoxifen as firstline therapy for advanced breast cancer in 668 postmenopausal women: results of the Tamoxifen or Arimidex Randomized Group Efficacy and Tolerability study. J Clin Oncol 2000; 18:3748-57.

4. Mouridsen H, Gershanovich M, Sun Y, et al. Superior efficacy of letrozole versus tamoxifen as first-line therapy for postmenopausal women with advanced breast cancer: results of a phase III study of the International Letrozole Breast Cancer Group. J Clin Oncol 2001; 19:2596-606.

5. Nabholtz JM, Buzdar A, Pollak M, et al. Anastrozole is superior to tamoxifen as first-line therapy for advanced breast cancer in postmenopausal women: results of a North American multicenter randomized trial. Arimidex Study Group. J Clin Oncol 2000; 18:3758-67.

6. Paridaens RJ, Dirix LY, Beex LV, et al. Phase III study comparing exemestane with tamoxifen as first-line hormonal treatment of metastatic breast cancer in postmenopausal women: the European Organisation for Research and Treatment of Cancer Breast Cancer Cooperative Group. J Clin Oncol 2008; 26:4883-90.

7. The ATAC Trialists' Group. Anastrozole alone or in combination with tamoxifen versus tamoxifen alone for adjuvant treatment of postmenopausal women with early breast cancer: first results of the ATAC randomised trial. Lancet 2002; 359:2131-9.

8. Coombes RC, Hall E, Gibson LJ, et al. A randomized trial of exemestane after two to three years of tamoxifen therapy in postmenopausal women with primary breast cancer. $N$ Engl J Med 2004; 350:1081-92.

9. The Breast International Group (BIG) 1-98 Collaborative Group. A comparison of letrozole and tamoxifen in postmenopausal women with early breast cancer. $N$ Engl J Med 2005; 353:2747-57.

10. Wakeling AE. Similarities and distinctions in the mode of action of different classes of antioestrogens. Endocr Relat Cancer 2000; 7:17-28.

11. Wakeling AE, Dukes M, Bowler J. A potent specific pure antiestrogen with clinical potential. Cancer Res 1991; 51:3867-73. 
12. Osborne CK, Wakeling A, Nicholson RI. Fulvestrant: an oestrogen receptor antagonist with a novel mechanism of action. Br J Cancer 2004; 90 (Suppl 1):S2-S6.

13. Robertson JFR, Howell A, Gorbunova VA, et al. Sensitivity to further endocrine therapy is retained following progression on first-line fulvestrant. Breast Cancer Res Treat 2005; 92:169-74.

14. Robertson JF, Lindemann J, Llombart-Cussac A, et al. Fulvestrant $500 \mathrm{mg}$ versus anastrozole $1 \mathrm{mg}$ for the first-line treatment of advanced breast cancer: follow-up analysis from the randomized 'FIRST' study. Breast Cancer Res Treat 2012; 136:503-11.

15. Johnston S. Fulvestrant and the sequential endocrine cascade for advanced breast cancer. Br J Cancer 2004; 90 (Suppl 1):S15-S18.

16. Robertson JF, Osborne CK, Howell A, et al. Fulvestrant versus anastrozole for the treatment of advanced breast carcinoma in postmenopausal women: a prospective combined analysis of two multicenter trials. Cancer 2003; 98:229-38.

17. Ingle JN, Suman VJ, Rowland KM, et al. Fulvestrant in women with advanced breast cancer after progression on prior aromatase inhibitor therapy: North Central Cancer Treatment Group trial N0032. J Clin Oncol 2006; 24:1052-6.

18. Chia S, Gradishar W, Mauriac L, et al. Double-blind, randomized placebo controlled trial of fulvestrant compared with exemestane after prior nonsteroidal aromatase inhibitor therapy in postmenopausal women with hormone receptor-positive, advanced breast cancer: results from EFECT. J Clin Oncol 2008; 26:1664-70.

19. Makris A, Powles TJ, Allred DC, et al. Changes in hormone receptors and proliferation markers in tamoxifen treated breast cancer patients and the relationship with response. Breast Cancer Res Treat 1998; 48:11-20.

20. Robertson JF, Nicholson RI, Bundred NJ, et al. Comparison of the short-term biological effects of 7alpha-[9-(4,4,5,5,5-pentafluoropentylsulfinyl)-nonyl] estra-1,3,5, (10)-triene-3,17beta-diol (Faslodex) versus tamoxifen in postmenopausal women with primary breast cancer. Cancer Res 2001; 61:6739-46.

21. Urruticoechea A, Smith IE, Dowsett M. Proliferation marker Ki-67 in early breast cancer. J Clin Oncol 2005; 23:7212-20.

22. Goncalves R, Ma C, Luo J, et al. Use of neoadjuvant data to design adjuvant endocrine therapy trials for breast cancer. Nat Rev Clin Oncol 2012; 9:223-9.

23. Howell A, Robertson JFR, Quaresma Albano J, et al. Fulvestrant, formerly ICI 182,780 , is as effective as anastrozole in postmenopausal women with advanced breast cancer progressing after prior endocrine treatment. J Clin Oncol 2002; 20:3396-403. 
24. Osborne CK, Pippen J, Jones SE, et al. Double-blind, randomized trial comparing the efficacy and tolerability of fulvestrant versus anastrozole in postmenopausal women with advanced breast cancer progressing on prior endocrine therapy: results of a North American trial. J Clin Oncol 2002; 20:3386-95.

25. Di Leo A, Jerusalem G, Petruzelka L, et al. Results of the CONFIRM Phase III trial comparing fulvestrant $250 \mathrm{mg}$ with fulvestrant $500 \mathrm{mg}$ in postmenopausal women with estrogen receptor-positive advanced breast cancer. J Clin Oncol 2010; 28:4594-600.

26. DeFriend DJ, Howell A, Nicholson RI, et al. Investigation of a new pure antiestrogen (ICI 182780) in women with primary breast cancer. Cancer Res 1994; 54:408-14.

27. Kuter I, Gee JM, Hegg R, et al. Dose-dependent change in biomarkers during neoadjuvant endocrine therapy with fulvestrant: results from NEWEST, a randomized Phase II study. Breast Cancer Res Treat 2012; 133:237-46.

28. Robertson JF, Dixon JM, Sibbering DM, et al. A randomized trial to assess the biological activity of short-term (pre-surgical) fulvestrant $500 \mathrm{mg}$ plus anastrozole versus fulvestrant $500 \mathrm{mg}$ alone or anastrozole alone on primary breast cancer. Breast Cancer Res 2013; 15:R18.

29. Di Leo A, Jerusalem G, Petruzelka L, et al. Final overall survival: fulvestrant 500mg vs 250mg in the randomized CONFIRM trial. J Natl Cancer Inst 2014; 106:djt337.

30. Howell A, Robertson JFR, Abram P, et al. Comparison of fulvestrant versus tamoxifen for the treatment of advanced breast cancer in postmenopausal women previously untreated with endocrine therapy: a multinational, double-blind, randomized trial. J Clin Oncol 2004; 22:1605-13.

31. Robertson JFR, Llombart A, Rolski J, et al. Activity of fulvestrant $500 \mathrm{mg}$ versus anastrozole $1 \mathrm{mg}$ as first-line treatment for advanced breast cancer: results from the FIRST study. J Clin Oncol 2009; 27:4530-5.

32. Robertson JFR, Gutteridge E, Cheung KL, et al. Oestrogen receptor expression in human breast cancer during long-term fulvestrant treatment. Proc Am Soc Clin Oncol 2004; 2311 .

33. Agrawal A, Robertson JF, Cheung KL, et al. Biological effects of fulvestrant on oestrogen receptor positive human breast cancer: short, medium and long-term effects based on sequential biopsies. Manuscript in preparation 2013.

34. Bergh J, Jönsson PE, Lidbrink EK, et al. FACT: an open-label randomized Phase III study of fulvestrant and anastrozole in combination compared with anastrozole alone as first-line therapy for patients with receptor-positive postmenopausal breast cancer. J Clin Oncol 2012; 30:1919-25. 
35. Mehta RS, Barlow WE, Albain KS, et al. Combination anastrozole and fulvestrant in metastatic breast cancer. N Engl J Med 2012; 367:435-44.

36. Johnston S, Kilburn LS, Ellis P, et al. Fulvestrant alone or with concomitant anastrozole vs exemestane following progression on non-steroidal aromatase inhibitor first results of the SoFEa Trial (CRUKE/03/021 \& CRUK/09/007) (ISRCTN44195747). Eur J Cancer 2012; 48 (Suppl 3):S2.

37. Robertson JFR, Semiglazov V, Nemsadze G, et al. Effects of fulvestrant $250 \mathrm{mg}$ in premenopausal women with oestrogen receptor-positive primary breast cancer. Eur $J$ Cancer 2007; 43:64-70.

38. Young OE, Renshaw L, Macaskill EJ, et al. Effects of fulvestrant $750 \mathrm{mg}$ in premenopausal women with oestrogen-receptor-positive primary breast cancer. Eur $J$ Cancer 2008; 44:391-9.

39. Robertson JF, Odling-Smee W, Holcombe C, et al. Pharmacokinetics of a single dose of fulvestrant prolonged-release intramuscular injection in postmenopausal women awaiting surgery for primary breast cancer. Clin Ther 2003; 25:1440-52.

40. Kuter I, Sapunar F, McCormack P. Pharmacokinetic profile of fulvestrant $500 \mathrm{mg}$ vs 250 mg: results from the NEWEST study. J Clin Oncol 2008; 26 (Suppl).

41. Robertson JF, Erikstein B, Osborne KC, et al. Pharmacokinetic profile of intramuscular fulvestrant in advanced breast cancer. Clin Pharmacokinet 2004; 43:52938.

42. McCormack P, Sapunar F. Pharmacokinetic profile of the fulvestrant loading dose regimen in postmenopausal women with hormone receptor-positive advanced breast cancer. Clin Breast Cancer 2008; 8:347-51.

43. Ohno S, Rai Y, Iwata H, et al. Three dose regimens of fulvestrant in postmenopausal Japanese women with advanced breast cancer: results from a double-blind, phase II comparative study (FINDER1). Ann Oncol 2010; 21:2342-7.

44. Pritchard KI, Rolski J, Papai Z, et al. Results of a phase II study comparing three dosing regimens of fulvestrant in postmenopausal women with advanced breast cancer (FINDER2). Breast Cancer Res Treat 2010; 123:453-61. 
Table 1. Change from baseline in ER H-score.

\begin{tabular}{|c|c|c|c|c|}
\hline \multirow[t]{2}{*}{ Treatment } & \multicolumn{4}{|c|}{$\begin{array}{l}\text { Back-transformed least squares mean change from baseline } \\
\qquad(\%) \\
(95 \% \mathrm{CI})\end{array}$} \\
\hline & Study 18 & NEWEST & $\begin{array}{c}\text { Trial } 57 \\
\text { initial phase }\end{array}$ & $\begin{array}{c}\text { Trial } 57 \\
\text { main phase }\end{array}$ \\
\hline Placebo & $\begin{array}{c}-37.3 \\
(-69.5,28.9)\end{array}$ & & & \\
\hline Tamoxifen & $\begin{array}{c}-61.7 \\
(-82.5,-15.9)\end{array}$ & & & \\
\hline Fulvestrant $50 \mathrm{mg}$ & $\begin{array}{c}-67.6 \\
(-83.7,-35.5)\end{array}$ & & & \\
\hline Fulvestrant $125 \mathrm{mg}$ & $\begin{array}{c}-75.2 \\
(-87.0,-52.4)\end{array}$ & & & \\
\hline Fulvestrant $250 \mathrm{mg}$ & $\begin{array}{c}-84.0 \\
(-91.7,-69.1)\end{array}$ & $\begin{array}{c}-10.7 \\
(-30.3,14.4)\end{array}$ & $\begin{array}{c}-21.0 \\
(-56.2,42.4)\end{array}$ & \\
\hline Fulvestrant $500 \mathrm{mg}$ & & $\begin{array}{c}-52.9 \\
(-63.0,-40.1)\end{array}$ & & $\begin{array}{c}-44.6 \\
(-53.9,-33.4)\end{array}$ \\
\hline $\begin{array}{l}\text { Fulvestrant } 250 \mathrm{mg} \text { plus } \\
\text { anastrozole }\end{array}$ & & & $\begin{array}{c}-43.2 \\
(-68.4,2.1)\end{array}$ & \\
\hline $\begin{array}{l}\text { Fulvestrant } 500 \mathrm{mg} \text { plus } \\
\text { anastrozole }\end{array}$ & & & & $\begin{array}{c}-48.9 \\
(-58.1,-37.6)\end{array}$ \\
\hline Anastrozole & & & $\begin{array}{c}5.8 \\
(-41.0,89.7)\end{array}$ & $\begin{array}{c}-14.7 \\
(-29.7,3.5)\end{array}$ \\
\hline
\end{tabular}

CI, confidence interval; ER, estrogen receptor 
Table 2. Change from baseline in PgR H-score.

\begin{tabular}{|c|c|c|c|c|}
\hline \multirow[t]{2}{*}{ Treatment } & \multicolumn{4}{|c|}{$\begin{array}{l}\text { Back-transformed least squares mean change from baseline } \\
\qquad(\%) \\
(95 \% \mathrm{CI})\end{array}$} \\
\hline & Study 18 & NEWEST & $\begin{array}{c}\text { Trial } 57 \\
\text { initial phase }\end{array}$ & $\begin{array}{c}\text { Trial } 57 \\
\text { main phase }\end{array}$ \\
\hline Placebo & $\begin{array}{c}40.3 \\
(-25.8,165.4)\end{array}$ & & & \\
\hline Tamoxifen & $\begin{array}{c}160.1 \\
(27.7,429.8)\end{array}$ & & & \\
\hline Fulvestrant $50 \mathrm{mg}$ & $\begin{array}{c}-62.7 \\
(-80.6,-28.6)\end{array}$ & & & \\
\hline Fulvestrant $125 \mathrm{mg}$ & $\begin{array}{c}-78.8 \\
(-88.4,-61.3)\end{array}$ & & & \\
\hline Fulvestrant $250 \mathrm{mg}$ & $\begin{array}{c}-86.4 \\
(-92.8,-74.2)\end{array}$ & $\begin{array}{c}-67.3 \\
(-81.0,-43.7)\end{array}$ & $\begin{array}{c}-47.5 \\
(-82.8,60.3)\end{array}$ & \\
\hline Fulvestrant $500 \mathrm{mg}$ & & $\begin{array}{c}-91.4 \\
(-95.0,-85.0)\end{array}$ & & $\begin{array}{c}-63.2 \\
(-77.2,-40.6)\end{array}$ \\
\hline $\begin{array}{l}\text { Fulvestrant } 250 \mathrm{mg} \text { plus } \\
\text { anastrozole }\end{array}$ & & & $\begin{array}{c}-49.2 \\
(-82.9,50.9)\end{array}$ & \\
\hline $\begin{array}{l}\text { Fulvestrant } 500 \mathrm{mg} \text { plus } \\
\text { anastrozole }\end{array}$ & & & & $\begin{array}{c}-58.3 \\
(-75.5,-29.0)\end{array}$ \\
\hline Anastrozole & & & $\begin{array}{c}-65.9 \\
(-88.8,4.0)\end{array}$ & $\begin{array}{c}-59.2 \\
(-75.0,-33.6)\end{array}$ \\
\hline
\end{tabular}

CI, confidence interval; PgR, progesterone receptor. 
Table 3. Change from baseline in Ki67 labeling index.

\begin{tabular}{|c|c|c|c|c|}
\hline \multirow[t]{2}{*}{ Treatment } & \multicolumn{4}{|c|}{$\begin{array}{l}\text { Back-transformed least squares mean change from baseline } \\
\qquad(\%) \\
(95 \% \mathrm{CI})\end{array}$} \\
\hline & Study 18 & NEWEST & $\begin{array}{c}\text { Trial } 57 \\
\text { initial phase }\end{array}$ & $\begin{array}{c}\text { Trial } 57 \\
\text { main phase }\end{array}$ \\
\hline Placebo & $\begin{array}{c}3.7 \\
(-18.0,31.1)\end{array}$ & & & \\
\hline Tamoxifen & $\begin{array}{c}35.8 \\
(-51.3,-15.5)\end{array}$ & & & \\
\hline Fulvestrant $50 \mathrm{mg}$ & $\begin{array}{c}-23.3 \\
(-40.6,-0.9)\end{array}$ & & & \\
\hline Fulvestrant $125 \mathrm{mg}$ & $\begin{array}{c}-46.1 \\
(-58.6,-29.7)\end{array}$ & & & \\
\hline Fulvestrant $250 \mathrm{mg}$ & $\begin{array}{c}-46.5 \\
(-58.1,-31.6)\end{array}$ & $\begin{array}{c}-45.5 \\
(-58.5,-28.2)\end{array}$ & $\begin{array}{c}-79.0 \\
(-90.4,-53.7)\end{array}$ & \\
\hline Fulvestrant $500 \mathrm{mg}$ & & $\begin{array}{c}-81.2 \\
(-85.8,-75.0)\end{array}$ & & $\begin{array}{c}-74.4 \\
(-81.5,-64.5)\end{array}$ \\
\hline $\begin{array}{l}\text { Fulvestrant } 250 \mathrm{mg} \text { plus } \\
\text { anastrozole }\end{array}$ & & & $\begin{array}{c}-91.1 \\
(-96.0,-80.2)\end{array}$ & \\
\hline $\begin{array}{l}\text { Fulvestrant } 500 \mathrm{mg} \text { plus } \\
\text { anastrozole }\end{array}$ & & & & $\begin{array}{c}-83.4 \\
(-88.5,-76.0)\end{array}$ \\
\hline Anastrozole & & & $\begin{array}{c}-84.4 \\
(-92.9,-65.6)\end{array}$ & $\begin{array}{c}-85.0 \\
(-89.1,-79.4)\end{array}$ \\
\hline
\end{tabular}

CI, confidence interval. 
Table 4. PFS in fulvestrant monotherapy trials for advanced breast cancer.

PFS (months)

$\begin{array}{lcccccc}\text { Study design } & \text { Fulvestrant } & \text { Fulvestrant } & \text { Fulvestrant } & \text { Tamoxifen } & \text { Anastrozole } & \text { Exemestane } \\ 250 \mathrm{mg} & 250 \mathrm{mg}+ & 500 \mathrm{mg} & 20 \mathrm{mg} / \text { day } & 1 \mathrm{mg} / \mathrm{day} & 25 \mathrm{mg} / \mathrm{day} \\ & \mathrm{LD} & & \end{array}$

\section{Second-line studies}

Study $20^{\mathrm{a}}$

Phase III, randomized, open-label,

fulvestrant $250 \mathrm{mg}, \mathrm{n}=222$

anastrozole, $\mathrm{n}=229^{23}$.

Study $21^{\mathrm{a}}$

Phase III, randomized, double-blind, parallel-group, double-dummy,

multicenter study: fulvestrant

$250 \mathrm{mg}, \mathrm{n}=206$; anastrozole, $\mathrm{n}=194$

24 .

EFECT Phase III, randomized, double-blind,

double-dummy, multicenter study:

fulvestrant $250 \mathrm{mg}+\mathrm{LD}, \mathrm{n}=351$;

exemestane, $\mathrm{n}=342^{18}$.

FINDER1 Phase II, randomized, double-blind, parallel-group, multicenter study: fulvestrant $500 \mathrm{mg}, \mathrm{n}=47$; fulvestrant $250 \mathrm{mg}+\mathrm{LD}, \mathrm{n}=51$; fulvestrant $250 \mathrm{mg}, \mathrm{n}=45^{43}$. 
FINDER2

\section{First-line studies}

\section{Trial 25}

Phase II, randomized, double-blind, parallel-group, multicenter study: fulvestrant $500 \mathrm{mg}, \mathrm{n}=46$; fulvestrant

$250 \mathrm{mg}+\mathrm{LD}, \mathrm{n}=51$; fulvestrant

$250 \mathrm{mg}, \mathrm{n}=47^{44}$

Phase III, randomized, double-blind, parallel-group, multicenter study:

fulvestrant $500 \mathrm{mg}, \mathrm{n}=362$;

fulvestrant $250 \mathrm{mg}, \mathrm{n}=374^{25}$.

Phase III, randomized, double-blind,

parallel-group, double-dummy,

multicenter study: fulvestrant

$250 \mathrm{mg}, \mathrm{n}=313$; tamoxifen, $\mathrm{n}=274^{30}$.

Phase II, randomized, open-label,

parallel-group, multicenter study:

fulvestrant $500 \mathrm{mg}, \mathrm{n}=102$;

anastrozole, $n=103^{14}$.

\footnotetext{
${ }^{a}$ Studies 0020 and 0021 initially included a fulvestrant $125 \mathrm{mg}$ treatment group that was withdrawn due to lack of clinical activity.

${ }^{\mathrm{b}}$ Data from the FIRST follow-up analysis.
}

Fulvestrant $250 \mathrm{mg}: 250 \mathrm{mg}$ Days 0 and 28, $250 \mathrm{mg} / \mathrm{month}$ thereafter; fulvestrant $250 \mathrm{mg}$ + LD: $500 \mathrm{mg}$ Day 0, $250 \mathrm{mg}$ Days 14 and 28 ,

$250 \mathrm{mg} / \mathrm{month}$ thereafter; fulvestrant $500 \mathrm{mg}$ : $500 \mathrm{mg}$ Days 0,14 and 28, $500 \mathrm{mg} / \mathrm{month}$ thereafter.

LD, loading dose; PFS, progression-free survival 


\section{Figure legends}

Figure 1. Change from baseline in ER expression.

Figure 2. Change from baseline in PgR expression.

Figure 3. Change from baseline in Ki67 labeling index. 
Figure 1. Change from baseline in ER expression.

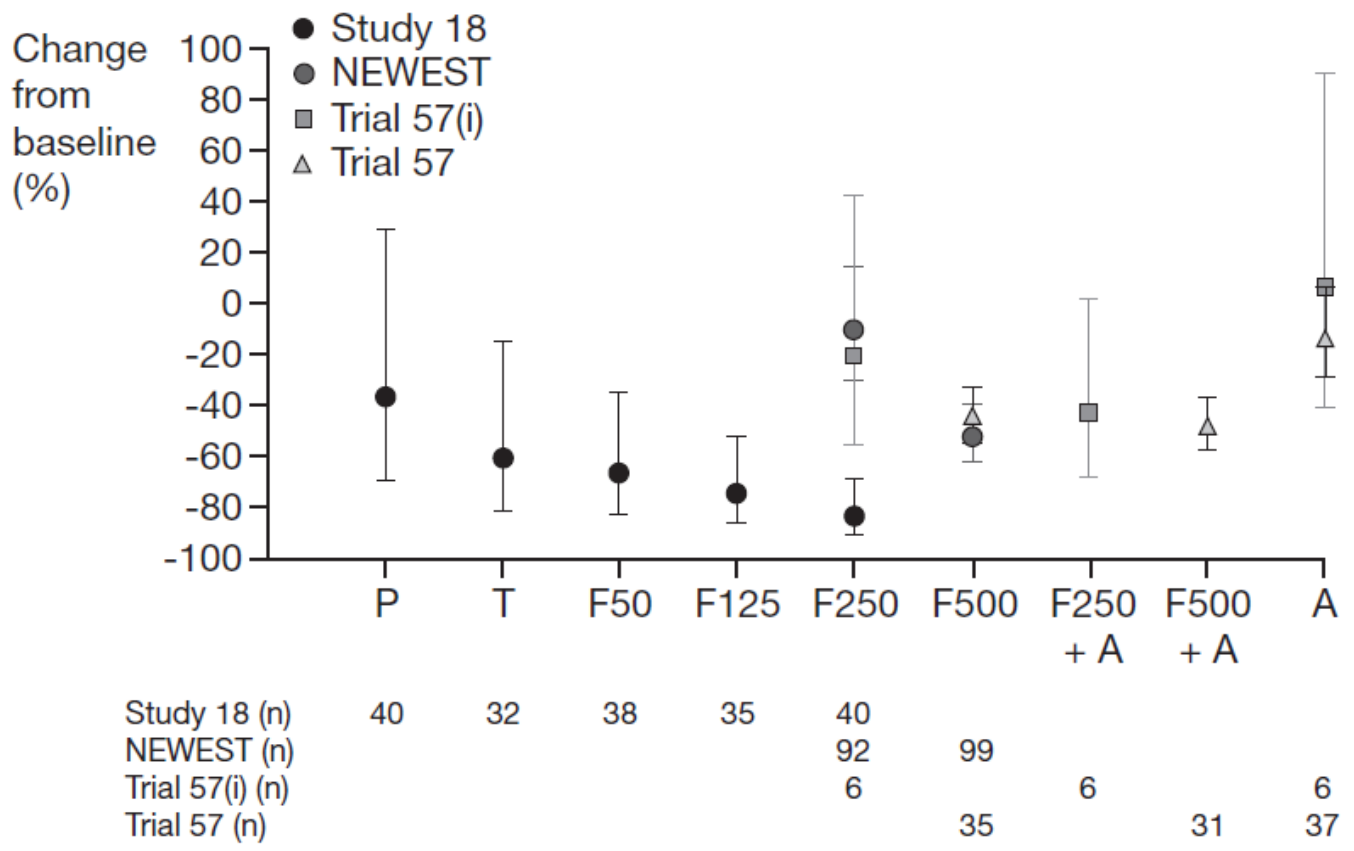

Least squares mean and $95 \%$ confidence interval; output from an analysis of covariance model of ER change from baseline (natural log transformed) with treatment as a factor.

Trial 57(i): data from initial patients in Trial 57, treated with F250, prior to protocol amendment. A, anastrozole; ER, estrogen receptor; F50/125/250/500, fulvestrant 50/125/250/500 mg; P, placebo; T, tamoxifen. 
Figure 2. Change from baseline in PgR expression.

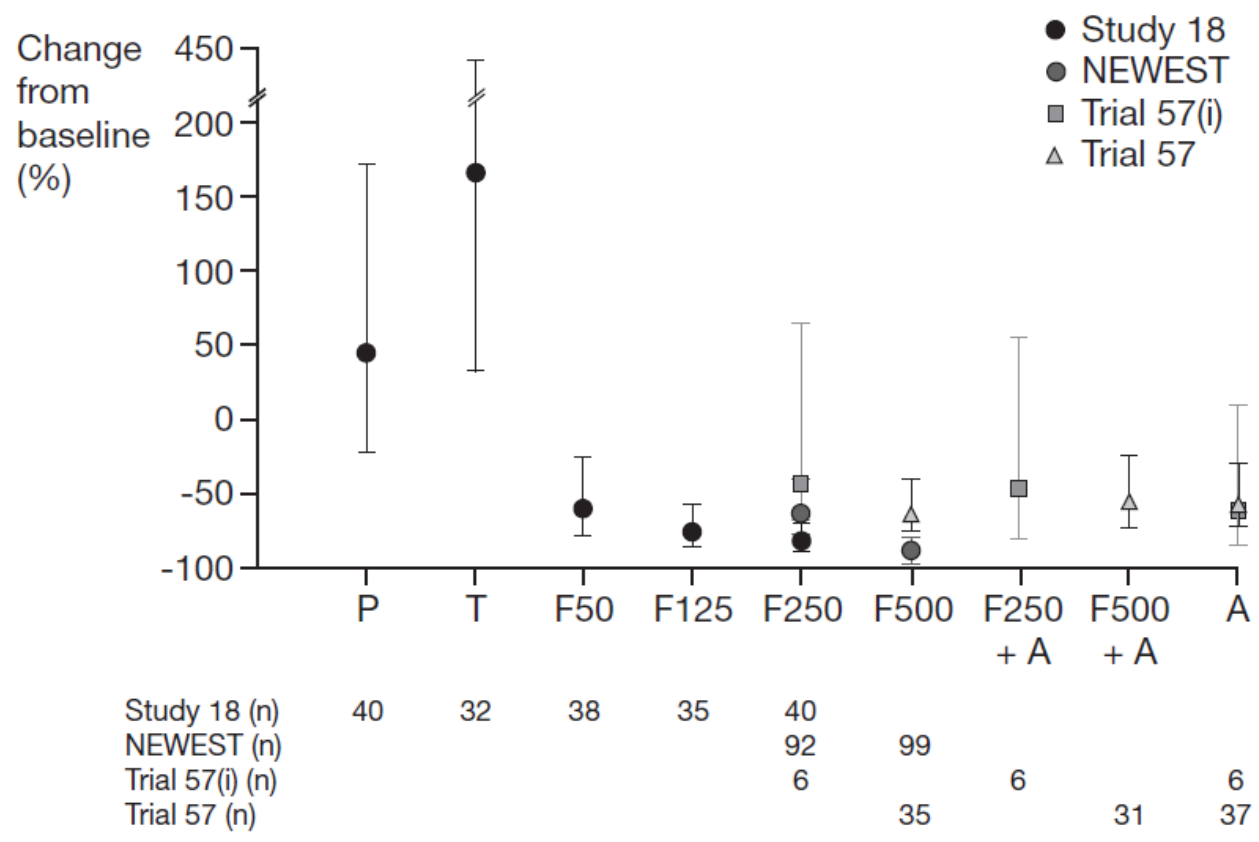

Least squares mean and 95\% confidence interval; output from an analysis of covariance model of PgR change from baseline (natural log transformed) with treatment as a factor.

Trial 57(i): data from initial patients in Trial 57, treated with F250, prior to protocol amendment.

A, anastrozole; F50/125/250/500, fulvestrant 50/125/250/500 mg; P, placebo; PgR, progesterone receptor; T, tamoxifen. 
Figure 3. Change from baseline in Ki67 labeling index.

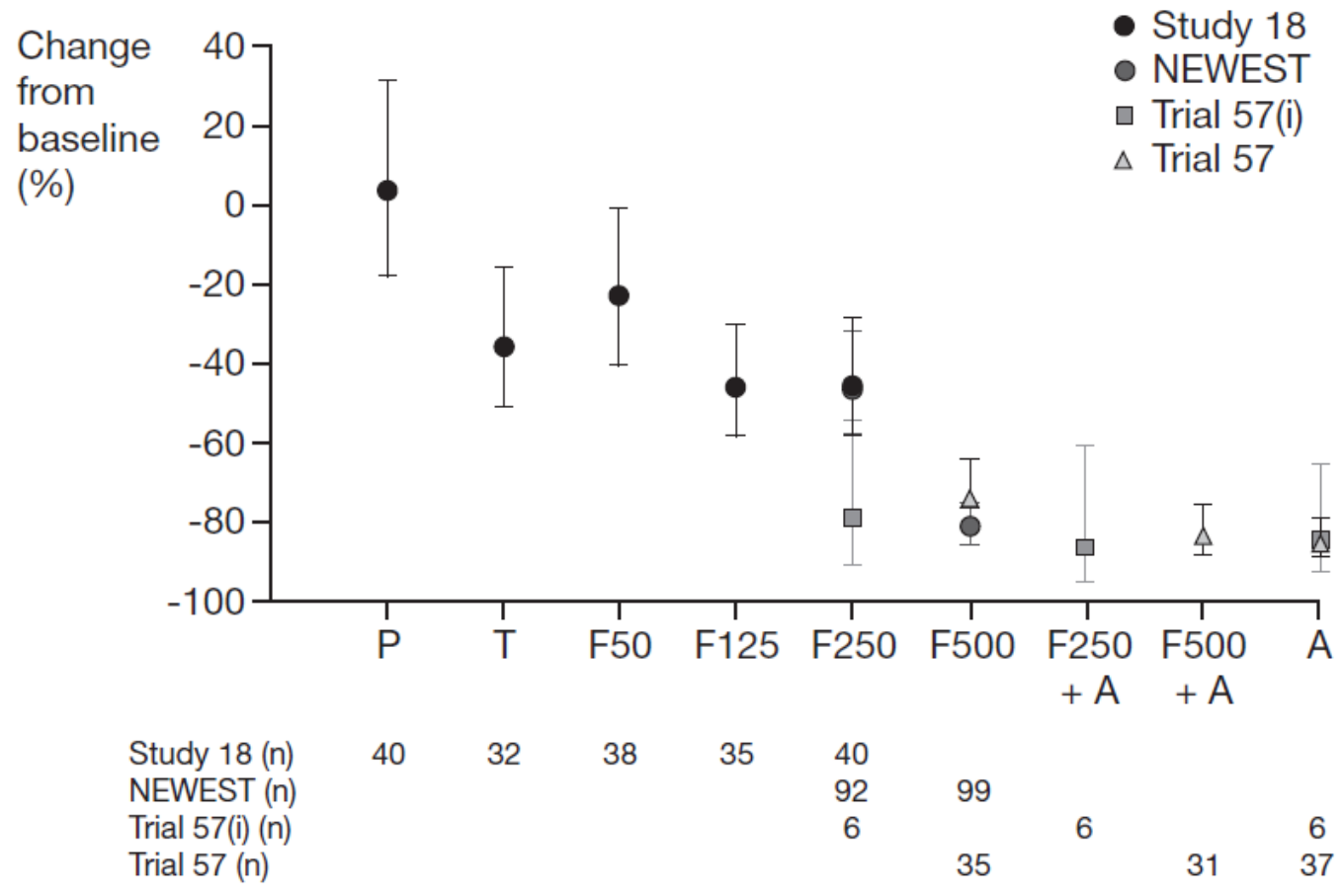

Least squares mean and 95\% confidence interval; output from an analysis of covariance model of Ki67 labelling index change from baseline (natural log transformed) with treatment as a factor. Trial 57(i): data from initial patients in Trial 57, treated with F250, prior to protocol amendment. A, anastrozole; F50/125/250/500, fulvestrant 50/125/250/500 mg; P, placebo; T, tamoxifen. 